\title{
Invasive mould infections in solid organ transplant patients: modifiers and indicators of disease and treatment response
}

\author{
Tobias Welte ${ }^{1}(0) \cdot$ Oscar Len ${ }^{2} \cdot$ Patricia Muñoz ${ }^{3,4} \cdot$ Luigina Romani $^{5} \cdot$ Russell Lewis $^{6} \cdot$ Alessandro Perrella $^{7,8}$
}

Received: 29 April 2019 / Accepted: 18 September 2019 / Published online: 1 October 2019

(c) The Author(s) 2019

\begin{abstract}
Purpose Invasive mould infections, in particular invasive aspergillosis (IA), are comparatively frequent complications of immunosuppression in patients undergoing solid organ transplantation (SOT). Guidelines provide recommendations as to the procedures to be carried out to diagnose and treat IA, but only limited advice for SOT recipients.

Methods Literature review and expert consensus summarising the existing evidence related to prophylaxis, diagnosis, treatment and assessment of response to IA and infections by Mucorales in SOT patients

Results Response to therapy should be assessed early and at regular intervals. No indications of improvement should lead to a prompt change of the antifungal treatment, to account for possible infections by Mucorales or other moulds such as Scedosporium. Imaging techniques, especially CT scan and possibly angiography carried out at regular intervals during early and long-term follow-up and coupled with a careful clinical diagnostic workout, should be evaluated as diagnostic tools and outcome predictors, and standardised to improve therapy monitoring. The role of biomarkers such as the galactomannan test and PCR, as well as selected inflammation parameters, has not yet been definitively assessed in the SOT population and needs to be studied further. The therapeutic workup should consider a reduction of immunosuppressive therapy.

Conclusions The role of immunosuppression and immune tolerance mechanisms in the response to invasive fungal infection treatment is an important factor in the SOT population and should not be underestimated. The choice of the antifungal should consider not only their toxicity but also their effects on the immune system, two features that are intertwined.
\end{abstract}

Keywords Aspergillus $\cdot$ Invasive pulmonary aspergillosis $\cdot$ Microbiome $\cdot$ Mucorales $\cdot$ Mucormycosis $\cdot$ Solid organ transplantation

\section{Introduction}

Invasive fungal infections (IFD) are comparatively frequent complications of immunosuppression in haemato-oncological patients, and, albeit to a lesser extent, in solid organ

Tobias Welte

welte.tobias@mh-hannover.de

1 Department of Respiratory Medicine, Hannover Medical School, Carl Neuberg Str 1, 30625 Hannover, Germany

2 Infectious Diseases Department, Hospital Universitari Vall d'Hebron, Barcelona, Spain

3 Department of Clinical Microbiology and Infectious Diseases, Hospital General Universitario Gregorio Marañón, Madrid, Spain

4 Medicine Department, Universidad Complutense de Madrid, CIBER Enfermedades Respiratorias-CIBERES (CB06/06/0058), Madrid, Spain transplant (SOT) recipients. Candida spp. are still the main aetiological agents (53\% in some studies) [1], followed by Aspergillus spp., Mucorales, and Cryptococcus [2]. The incidence of Aspergillus spp. in transplant patients ranges from 0.1 to $3.5 \%$, depending on the country and the type

5 Department of Experimental Medicine, School of Medicine, University of Perugia, 06132 Perugia, Italy

6 Infectious Diseases Hospital, S. Orsola-Malpighi, Department of Medical and Surgical Sciences, University of Bologna, Bologna, Italy

7 VII, Department of Infectious Disease and Immunology, Hospital D. Cotugno, Naples, Italy

8 CLSE-Liver Transplant Unit, Hospital A. Cardarelli, Naples, Italy 
Fig. 1 Balance between immunity and infection

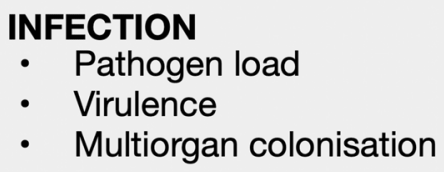

\section{IMMUNOSUPPRESSION}
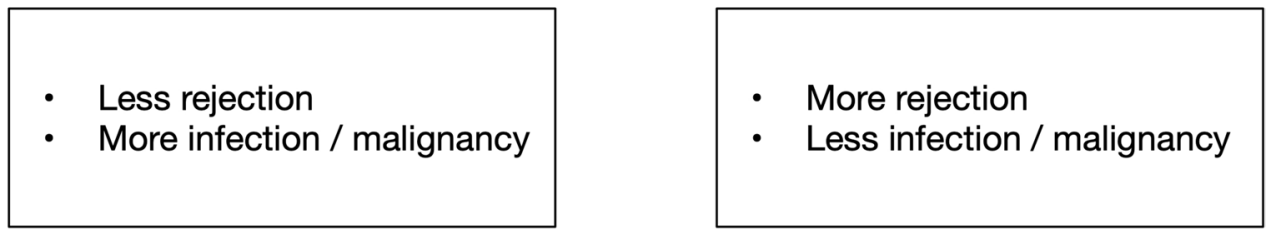

of transplant [2], invasive aspergillosis (IA) being most frequent among lung transplant patients, in whom it may emerge as a complication not only immediately after transplant but also at later stages [3]. Infection rates by Mисorales have been reported to be as high as 3\% [2-4], whereas Cryptococcus incidence can reach 1.5\% [2]. IFDs in SOT patients should be promptly diagnosed and treated, because they often lead to graft loss and death [5]. Mortality rates are high for all IFD, but in particular for mould infections, reaching $67-82 \%$ in patients with invasive pulmonary aspergillosis and $73 \%$ in cerebral forms of mucormycosis [2].

Patients with early graft rejection or graft dysfunction, or active or latent infection in the donor or recipient at the time of transplantation, are at particularly high risk to develop opportunistic infections [6]. In these patients, the intensity of immunosuppression, as well as cytomegalovirus (CMV) co-infection are the main drivers of IA (Fig. 1) [7]. Corticosteroids and other immunosuppressive agents used in SOT not only reduce the risk of organ rejection, but also increase the susceptibility of transplanted patients to infections by several opportunistic and obligate pathogens (Nocardia, Pneumocystis, tuberculosis, and fungi) [8-12]. SOT recipients are also exposed to risk factors characteristic for each organ transplant population [13, 14]. Single lung transplantation and colonisation with Aspergillus spp. prior to transplantation procedure in lung transplant recipients [15]; bloodstream infections, pre-transplant chronic pulmonary obstructive disease, impaired graft function, long-term dialysis prior to transplantation, and serious post-transplant infections in kidney transplant patients [16, 17]; MELD score, choledochojejunostomy anastomosis, bacterial infections in the first month and absence of antifungal prophylaxis [18], CMV reactivation [17], renal failure, hemodialysis [3], and re-transplantation or transplantation for fulminant hepatic failure or reoperation $[19,20]$ in liver transplant recipients tend to increase the likelihood of IFD, in particular IA.
Animal studies have shown that the type of immunosuppressive regime strongly influences host responses and pathology to invasive pulmonary aspergillosis. In the setting of chemotherapy-associated neutropenia, both pro- and anti-inflammatory cytokine responses are induced following challenge with Aspergillus spores with increased TNF- $\alpha$ and IL-10 production, whereas immunosuppression with corticosteroids diminishes pro-inflammatory cytokine and IL-10 production following spore inhalation, with extensive polymorphonuclear neutrophil (PMN) recruitment in the tissue [21]. In neutropenic animals, tissue damage in the lung was driven primarily by fungal proliferation, which could be reduced with amphotericin B. In corticosteroid-immunosuppressed animals, however, extensive PMN infiltration contributes to tissue damage and amphotericin B is ineffective at prolonging survival [21]. The host defence mechanisms against Aspergillus are influenced by innate and adaptive immune signalling [22], and a thorough understanding of the immunological processes underlying transplantation is likely to improve outcomes and reduce the burden of opportunistic infections, as well as to minimise long-term toxicity of immunosuppression [23].

Existing guidelines provide general recommendations as to the procedures to be carried out to diagnose and treat IA [7, 24, 25]. The ESCMID 2017 Executive Summary [25] provides general recommendations for the diagnosis of IA in neutropenic and non-neutropenic patients but gives only limited advice about SOT recipients. The International Society for Heart and Lung Transplantation (ISHLT) Guidelines offers more specific information [26], but the overall evidence available for IA diagnosis and therapy in SOT recipients is scant. Assessment of response to pharmacological IA treatment in SOT recipients is also not specifically discussed in current treatment guidelines.

This brief review aims at summarising the existing evidence related to prophylaxis, diagnosis, treatment and 
assessment of early response to invasive mould infections (IMI), in particular IA, in SOT recipients.

\section{Epidemiology and diagnosis of IMI}

Spores circulating in the air are the main infection source of IA, not only in lung but also in other transplant recipients; thus, the presence of Aspergillus spores in the air of ICU and transplant units should be closely monitored [27].

Pulmonary complications are common occurrences in SOT patients, regardless of their aetiology [28]. Pulmonary infiltrates, nodular lesions, and pleural effusion have been reported in heart transplant patients with IA [27], although widespread pulmonary infiltrates may also be caused by primary graft dysfunction (PGD) [8]. In lung transplant recipients, chronic lung allograft dysfunction (CLAD) may also increase the risk of IA [29]. Overall, IA should always be suspected when pulmonary lesions are present, the lung often being the primary site of disease [30].

Imaging plays a major role in the diagnostic workout of SOT patients suspected of IA infection. In patients with haematological malignancies, the presence of angioinvasive lesions detected by computed tomography (CT) such as halo sign, nodules without halo, or later signs including air-crescent signs, and new cavities, are suggestive but not specific for Aspergillus or other mould infections [31]. In non-neutropenic patients, on the other hand, airway-invasive forms (small airway lesions, peribronchial consolidation, large nodules, ground-glass opacities) are more common at the time of initial CT $[32,33]$. Cavitated nodules and alveolar infiltrates, often with bilateral pulmonary involvement, are frequent in early and late episodes of IA [30, 34 ], and $37 \%$ of invasive pulmonary aspergillosis in heart transplant recipients present airway-invasive radiological pattern [30]. In patients with haematological malignancies, computed tomography pulmonary angiography (CTPA) has been shown to detect vessel occlusion sign (VOS) [35, 36], which provides direct evidence of an angioinvasive process. Recently, Henzler et al. [37] suggested that CTPA may also be a very sensitive and specific radiological test for SOT recipients, as VOS in non-neutropenic patients was the strongest radiological predictor of IA.

The ESCMID 2017 executive summary [25] recommends cultures and Aspergillus species identification for any patient population. In fact, cultures from sputum, BAS, BAL, CTguided transthoracic biopsies, video-assisted thoracoscopic surgery, open lung biopsies, transbronchial biopsies or convex endobronchial ultrasound transbronchial needle aspiration are often attempted, but their success is limited [38]. No specific data are available for SOT recipients, although some published work included also this patient population in the collectives studied [39]. Overall, however, sensitivity and specificity values of culture and microscopy work are rather modest also in SOT patients [25], improving only after resampling.

The beta-D-glucan (BDG) test is not specific for Aspergillus and in addition, its specificity in BAL is poor [40]. BDG is often used as a first-stage screening test, being present in large amounts in the cell wall of several fungal species, including Candida and Pneumocystis [41, 42], exceptions being Cryptococcus and Mucorales [42]. According to Koo et al. [43], a positive BDG test increases the diagnostic probability of IFD to approximately $50 \%$ and indicates the need for more extensive and possibly invasive diagnostic procedures. No specific data are available, however, for SOT recipients.

The galactomannan (GM) test has been shown to be highly reliable to diagnose IA in haemato-oncological patients [44-46]. This is not the case for the SOT population: in lung transplant patients, the GM test on bronchoalveolar lavage (BAL) samples, at a cut-off value of 0.5 , provided a higher specificity than a pan-Aspergillus PCR $(92 \%$ vs. $50 \%$ ), with mould-active prophylaxis having only little impact on performance [47], but Miceli et al. [48] reported a large percentage (51\%) of false-positive GM tests among lung and non-lung transplant recipients. The current IDSA guidelines do not recommend the use of the GM test for screening purposes in SOT patients [7]. The poorer sensitivity of serum GM in non-neutropenic patients likely reflects the lower frequency of angioinvasive disease in the lung at the time of testing, as well as host-mediated effects (i.e. PMN-mediated GM antigen clearance) [49]. The GM test of BAL samples, on the other hand, provides a more accurate and reliable picture of a potential IA in SOT recipients [19, 50].

PCR has been shown to be useful to detect and identify Aspergillus and other moulds, including Mucorales, in plasma, serum and BAL of haematological patients [51, 52], with higher sensitivity values obtained from BAL than from blood samples $[52,53]$. Only scant data are available for SOT patients, but Luong et al. [47] described very good sensitivity (100\%) and specificity (93\%) values for a PCR carried out on BAL samples of lung transplant recipients. The usefulness of PCR to detect IA in patients with no haematological cancer was also confirmed for a commercial PCR assay [54].

The combination of several biomarkers to improve diagnostic sensitivity and specificity has been advocated by several authors. Hasseine et al. [55] included 313 haematooncological and SOT patients in a multicentre, prospective study aiming at evaluating the performance of different strategies to diagnose invasive fungal infections. They concluded that the combination of different biomarkers and test, combined with CT scan results, could be beneficial to improve diagnostic specificity, sensitivity and, in 
particular, negative predictive values. Other authors have recommended similar strategies, combining results from the lateral-flow device (LFD) test and PCR [56], LFD and GM tests [48], and PCR and GM test [44, 45, 57]. These studies included only few SOT patients, but the results suggest that the combined use of several biomarkers and clinical parameters could provide a better diagnostic power also in SOT patients with suspected IMI. Possibly, the use of inflammation parameters such as those discussed by Heinz et al. [58] could further refine the diagnostic performance in SOT patients.

\section{Prophylaxis}

Recommendations for primary antifungal prophylaxis in SOT recipients vary mainly across different transplant centres $[29,59,60]$. The ISHLT Guidelines for the management of fungal infections in mechanical circulatory support and cardiothoracic organ transplant recipients include a summary of recommendations for prophylaxis in adults and provide some considerations on its use in paediatric transplant patients [13]. The ESCMID 2017 Executive Summary on the diagnosis and management of Aspergillus diseases contains a description of the factors that place SOT recipients at high risk of infections and recommends considering universal prophylaxis for lung transplant patients and targeted prophylaxis in the remaining SOT groups [25], while the 2016 IDSA Guidelines advise the adoption of a scheme based on the institutional epidemiology of infection and assessment of individual risk [7]. The different approach provided by both guidelines and the lack of binding recommendations for the antifungals to be used reflect not only the lack of evidence-based data but also the variability in policies at national and continental level [24].

For heart transplant recipients, Muñoz et al. [34] recommend a targeted IA prophylaxis for patients with at least one risk factor for infection. To prevent invasive aspergillosis, the ESCMID-ECMM_ERS Guideline recommends inhaled $\mathrm{AmB}$ or voriconazole in lung recipients, itraconazole or inhaled AmB in heart recipients, and AmB and echinocandins in liver recipients [25]. Nebulised amphotericin B has been successfully used to prevent Aspergillus spp. infections, particularly in patients with CLAD [29], as well as in liver transplant patients $[59,60]$. Long-term prophylaxis, particularly in institutions with significant air contamination, can significantly reduce IA incidence and mortality [34]. Overall, however, evidence-based information on the pharmacological prevention of IA in SOT patients is still lacking.

\section{Treatment}

In SOT patients, consideration of the extent of immunosuppression and of the transplanted organ, should guide prophylaxis and treatment of IMI. Azoles, amphotericin $\mathrm{B}$, and echinocandins all have the potential to interact with immunomodulatory drugs [61], and a therapeutic drug monitoring (TDM) may be required for triazole antifungals because of their variable pharmacokinetic properties and increased potential for pharmacokinetic drug-drug interactions [62].

Voriconazole is considered as the first-line therapy for the treatment of invasive aspergillosis [24, 63-65] and liposomal amphotericin B for mucormycosis in the Global guideline for the diagnosis and management of mucormycosis [66] presently under public consultation. Voriconazole is still considered as the first-line treatment for Scedosporium [67]. For infections by other hyalohyphomycoses, treatment has not yet been established [67]. Isavuconazole is apparently as effective as voriconazole in the treatment of IA and is characterised by a reduced liver toxicity, but the pivotal clinical trial included only haemato-oncological patients [68]. The ESCMID-ECMMERS guidelines recommend the use of echinocandins only in a combination therapy of azole-resistant Aspergilli [25]. Recommendations for treatment of adult cardiothoracic transplant candidates and recipients are included in the ISHLT guidelines [13], which also discuss therapeutic strategies in the paediatric population, and in the AST Infectious Diseases Community of Practice [19]. Some information is also provided by the ESCMID 2017 executive summary [25]. Overall, however, most evidence for the treatment and prevention of IMI in SOT patients is still based on clinical experience [2]. Because of the apparently only mild drug-drug interactions [69], the new triazole isavuconazole has the potential to become an important addition to the antifungal armamentarium, but specific studies are still needed to validate its use in SOT patients [70].

As with patients with haematological malignancies, the role of combination antifungal therapy is still controversial. Combination therapy is most frequently considered when no single drug provides sufficient protection, triazole drug exposures are unknown (i.e. while awaiting TDM results), or the patients present with breakthrough fungal infections [25, 71]. Nebulised amphotericin B therapy has been used as an adjunct to a primary voriconazole antifungal therapy to treat a mixed infection by A. fumigatus, Scedosporium prolificans, and Candida glabrata [72]. The ISHLT guidelines, however, underline the lack of welldesigned, randomised trials evaluating combination therapies in the treatment of IFD in SOT patients [26]. 
Table 1 Diagnostic and therapeutic challenges in SOT recipients

Heterogeneous population
Patients with different types of transplant
Different levels of immunosuppression depending on timing after
SOT
Diagnostic challenges
Difficulty to distinguish colonization from infection
Diagnostic tests less reliable (GM)
CT criteria made for neutropenic patients, less well defined for SOT
recipients
Drug interactions
Voriconazole-sirolimus
Azoles-tacrolimus
Drug toxicity
Azoles: liver toxicity
Amphotericin B: kidney toxicity

\section{Evaluation of treatment response}

For neutropenic patients, the Mycoses Study Group (MSG) and European Organization for Research and Treatment of Cancer (EORTC) has established definitions of success as a partial or complete response to therapy, and of failure as a stable response, disease progression, or death [73]. These definitions apply also to the SOT population, which, however, is more heterogeneous. SOT patients receive different types of transplants, undergo different levels of immunosuppression depending on the time after SOT, and are exposed to significant drug-drug interactions and drug toxicities (Table 1). Diagnostic tests such as GM are less reliable [47, $48,74]$, and the CT criteria used in the neutropenic population are less well defined. In fact, almost no information exists on the use of imaging and biomarkers as indicators of response success after treatment of IMI in SOT patients.

In neutropenic patients, CT scan results of improvement seem to be closely correlated with IA treatment success [75, 76]. Studies analysing initial and follow-up CT findings in SOT recipients with invasive pulmonary aspergillosis have shown that the presence of small $\left(<3 \mathrm{~cm}^{2}\right)$ nodules as well as the incidence of small cavities may be associated with better prognosis [32]. Lim et al. [32] also observed that, in SOT recipients, $83 \%$ of nodules remained unchanged or increased in size after 7 days and the mean mass reduction after 21 days was nearly 57\%. In haemato-oncological patients, Vehreschild et al. [77] have observed that small cavities are usually associated with a favourable prognosis, even if CT signs may take weeks or months to completely normalise. CT volumetry, therefore, seems a promising approach to establish criteria defining therapy response in neutropenic patients and should be further explored in the SOT population. The ESCMID Study Group for Infections in Compromised Hosts recommends to monitor the therapeutic response in adult SOT patients by clinical follow-up, with periodical high-resolution CT every 7-10 days during the first weeks of therapy in adults [2], a proposal that has also been put forward by Heinz et al. [58] for haematooncological patients.

With regards to biomarkers, a decrease of BDG levels during antifungal therapy is indicative of treatment response in candidemia [78], but the BDG test is not specific for Aspergillus and, therefore, it is no satisfactory outcome predictor in IA [7]. The GM test, on the other hand, has been shown to be a good prognostic factor of successful IA therapy in haematological patients [79-84]. In SOT patients, high GM indices (cut-off $\geq 2$ ) at diagnosis are indicative of a potentially poor outcome [85], but overall, the GM test is not as reliable as in neutropenic patients [47, 48, 86]. Increasing values are a concern for progressing infection and should prompt re-evaluation of antifungal therapy and/ or dosage escalation. Decreasing values are reassuring but should always be interpreted in the context of other radiological, laboratory and clinical signs of response.

Cytokines (serum interleukin (IL)-6, IL-8, IL-10, interferon- $\gamma$ ) [87], CRP [88], as well as haptoglobin (Hp) and annexin A1 (ANXA1) [89], have been proposed as potential immunological biomarker for assessing treatment response in patients with haematological malignancies [58]. All studies carried out so far [87, 90, 91], however, included only haemato-oncological patients. Their value in SOT patients is not established and interpretation may be confounded by a wide variety of factors typical of SOT recipients.

\section{Discussion}

The optimal approach to antifungal prophylaxis and treatment of IMI in SOT recipients is still unresolved. In this heterogeneous population, the individual immunological conditions seem to affect profoundly not only the outcome but also the results of routine clinical tests $[47,48,86]$. CT imaging, on the other hand, while presenting patterns that are distinct from those observed in haematological patients [32], seems to offer a comparatively reliable means to assess treatment response. In SOT patients, however, clinical judgement is even more crucial to guide therapeutic decisions than in haematological settings. Lack of improvement should prompt suspicion not only for a lack of response but also for infection by other mould species (Mucorales, Scedosporium) and lead to a quick change of therapeutic agent.

Voriconazole is still the first-line treatment of IA, with isavuconazole becoming a valuable alternative, but more options are needed. In this context, the use of liposomal antifungal formulations may prove to be a novel approach to fighting IMI. Empty liposomes have been shown in vivo to be anti-inflammatory immune modifiers $[92,93]$. Therefore, 
the use of liposomal formulations of active antifungals could, at least theoretically, help to improve the immune system of immunosuppressed patients. In high-risk SOT patients, the number of IFDs and even bacterial infections appeared to be reduced $[59,60]$, possibly by modulating IL-10 production before a strong antigen stimulation occurs [59], and a recent translational study has shown that L-AmB apparently cross Candida and Cryptococcus fungal walls as intact liposome vesicles, the crossing being facilitated by AmB [94]. Unfortunately, data on filamentous fungi are still lacking. This may explain the low toxicity of L-AmB and suggests a role of liposomal compounds in the transport and delivery of membrane-bound molecules to the fungal extracellular space [94]. In addition, Dectin-1 coating of L-AmB liposomes has shown enhanced efficacy in vitro, suggesting that coating of drug-containing liposomes with Dectin-1 could improve antifungal therapeutics [95].

Little attention has so far been devoted to the use of a pharmacokinetic/pharmacodynamic (PK/PD) approach to assess treatment response. Pharmacometrics [96] are now being used to re-analyse older data and develop a better scientific approach for future clinical trials with antibiotics. In antifungal therapy, biomarker-based PK/PD endpoints should also be explored and validated as a more targeted assessment of response to antifungal therapy in clinical settings.

Finally, the role of the patient microbiome in the development of and protection against fungal infections needs to be taken into consideration. In the last few years, for instance, a link between the presence of Fusobacterium nucleatum in the gut and the resistance to chemotherapy in colorectal cancer has been observed [97]. With regards to fungi, an aryl hydrocarbon receptor (AhR) activation by a Lactobacillus-derived AhR ligand has been shown to induce IL-22 production, thus activating mucosal defence mechanisms required for protection from fungal infections [98]. Changes in the microbiota composition may lead to the onset of fungal infections in different organs, caused by changes in the immune system of the host [99]. A better understanding of the host-microbiota system and its modifying agents may lead to improved therapeutic approaches and possibly discovery of new biomarkers that will help in better defining the assessment of a therapeutic response to fungal infections.

\section{Conclusions}

In all patients with IMI, antifungal therapy is crucial to reduce the high mortality rates associated with the infectious disease. Response to therapy should be assessed early and at regular intervals. No indications of improvement (minor or no improvement, or progressive infection) according to selected criteria should lead to a prompt change of the antifungal treatment, to cater for possible infections by Mucorales or other moulds such as Scedosporium. Imaging techniques such as CT scan and possibly angiography at regular intervals during early and long-term follow-up, coupled with a careful clinical diagnostic workout, should be evaluated for their use in SOT recipients with suspected IA as well as outcome predictors and should be standardised to improve therapy monitoring. PCR, galactomannan and beta-D-glucan tests, and selected inflammation parameters such as cytokines, are useful to assess treatment response in haematological patients. Their use in the SOT population, however, has not yet been definitively assessed, but these markers may provide additional information to guide clinical decisions.

The therapeutic workup should consider also a reduction of immunosuppressive therapy. Decreasing immunosuppression is a cornerstone in the treatment of SOT recipients with suspected IMI [23]. IFD are mainly a consequence of immunosuppression [5], particularly after the strong inflammatory response elicited by corticosteroids [61]. Thus, the role of immunosuppression and immune tolerance mechanisms in the response to IMI treatment should not be underestimated. For instance, the reduced infusion-related toxicity of liposomal amphotericin B (L-AmB) seems to be related to its effects on molecular immunological patterns [92], and the $\mathrm{AmB}$ deoxycholate toxicity linked to the production of pro-inflammatory cytokines [100]. Thus, the choice of the antifungal drug should be driven primarily by its antifungal activity, but its toxicity and its effects on the immune system, two features that are intertwined, should also be given the necessary attention [61].

Acknowledgements This work is the outcome of an expert meeting supported by an unrestricted grant by Gilead Sciences GmbH.

\section{Compliance with ethical standards}

Conflict of interest The authors declare that they have no competing interests.

Open Access This article is distributed under the terms of the Creative Commons Attribution 4.0 International License (http://creativeco mmons.org/licenses/by/4.0/), which permits unrestricted use, distribution, and reproduction in any medium, provided you give appropriate credit to the original author(s) and the source, provide a link to the Creative Commons license, and indicate if changes were made.

\section{References}

1. Pappas PG, Alexander BD, Andes DR, et al. Invasive fungal infections among organ transplant recipients: results of the Transplant-Associated Infection Surveillance Network (TRANSNET). Clin Infect Dis. 2010;50:1101-11. 
2. Gavaldà $J$, Meije Y, Fortún J, et al. Invasive fungal infections in solid organ transplant recipients. Clin Microbiol Infect. 2014;20:27-48.

3. Gavaldà J, Len $\mathrm{O}$, San Juan R, et al. Risk factors for invasive aspergillosis in solid-organ transplant recipients: a case-control study. Clin Infect Dis. 2005;41:52-9.

4. Cuenca-Estrella M, Bernal-Martinez L, Isla G, et al. Incidence of zygomycosis in transplant recipients. Clin Microbiol Infect. 2009;15:37-40.

5. Farmakiotis D, Kontoyiannis DP. Emerging issues with diagnosis and management of fungal infections in solid organ transplant recipients. Am J Transpl. 2015;15:1141-7.

6. Fishman JA. Infection in solid-organ transplant recipients. N Engl J Med. 2007;357:2601-14.

7. Patterson TF, Thompson GR 3rd, Denning DW, et al. Practice guidelines for the diagnosis and management of aspergillosis: 2016 update by the Infectious Diseases Society of America. Clin Infect Dis. 2016;63:e1-60.

8. Diamond JM, Lee JC, Kawut SM, et al. Clinical risk factors for primary graft dysfunction after lung transplantation. Am J Respir Crit Care Med. 2013;187:527-34.

9. Iriart X, Challan Belval T, Fillaux J, et al. Risk factors of Pneumocystis pneumonia in solid organ recipients in the era of the common use of posttransplantation prophylaxis. Am J Transpl. 2015;15:190-9.

10. Peleg AY, Husain S, Qureshi ZA, et al. Risk factors, clinical characteristics, and outcome of Nocardia infection in organ transplant recipients: a matched case-control study. Clin Infect Dis. 2007;44:1307-14.

11. Rello J, Bello I, de Vicente R, et al. Risk factors for mortality in 272 patients with lung transplant: a multicenter analysis of 7 intensive care units. Arch Bronconeumol. 2017;53:421-6.

12. Torre-Cisneros J, Doblas A, Aguado JM, et al. Tuberculosis after solid-organ transplant: incidence, risk factors, and clinical characteristics in the RESITRA (Spanish Network of Infection in Transplantation) cohort. Clin Infect Dis. 2009;48:1657-65.

13. Husain S, Camargo JF. Invasive aspergillosis in solid organ transplant recipients: guidelines from the American Society of Transplantation Infectious Diseases community of practice. Clin Transpl. 2019. https://doi.org/10.1111/ctr.13544.

14. Husain S, Silveira FP, Azie N, et al. Epidemiological features of invasive mold infections among solid organ transplant recipients: PATH Alliance(R) registry analysis. Med Mycol. 2017;55:269-77.

15. Aguilar CA, Hamandi B, Fegbeutel C, et al. Clinical risk factors for invasive aspergillosis in lung transplant recipients: results of an international cohort study. J Heart Lung Transpl. 2018;37:1226-34.

16. Lopez-Medrano F, Silva JT, Fernandez-Ruiz M, et al. Risk factors associated with early invasive pulmonary aspergillosis in kidney transplant recipients: results from a multinational matched case-control study. Am J Transpl. 2016;16:2148-57.

17. Rosenhagen M, Feldhues R, Schmidt J, et al. A risk profile for invasive aspergillosis in liver transplant recipients. Infection. 2009;37:313-9.

18. Saliba F, Delvart V, Ichai $P$, et al. Fungal infections after liver transplantation: outcomes and risk factors revisited in the MELD era. Clin Transpl. 2013;27:E454-61.

19. Singh N, Husain S. Aspergillosis in solid organ transplantation. Am J Transpl. 2013;13:228-41.

20. Winston DJ, Limaye AP, Pelletier S, et al. Randomized, double-blind trial of anidulafungin versus fluconazole for prophylaxis of invasive fungal infections in high-risk liver transplant recipients. Am J Transpl. 2014;14:2758-64.

21. Balloy V, Huerre M, Latgé J-P, et al. Differences in patterns of infection and inflammation for corticosteroid treatment and chemotherapy in experimental invasive pulmonary aspergillosis. Infect Immun. 2005;73:494-503.

22. van de Veerdonk FL, Gresnigt MS, Romani L, et al. Aspergillus fumigatus morphology and dynamic host interactions. Nat Rev Microbiol. 2017; 15:661-74.

23. Rosen HR. Transplantation immunology: what the clinician needs to know for immunotherapy. Gastroenterology. 2008;134:1789-801.

24. Garcia-Vidal C, Alastruey-Izquierdo A, Aguilar-Guisado M, et al. Executive summary of clinical practice guideline for the management of invasive diseases caused by Aspergillus: 2018 update by the GEMICOMED-SEIMC/REIPI. Enferm Infecc Microbiol Clin. 2018. https://doi.org/10.1016/j.eimc.2018.03.018.

25. Ullmann AJ, Aguado JM, Arikan-Akdagli S, et al. Diagnosis and management of Aspergillus diseases: executive summary of the 2017 ESCMID-ECMM-ERS guideline. Clin Microbiol Infect. 2018;24:e1-38.

26. Husain S, Sole A, Alexander BD, et al. The 2015 International Society for Heart and Lung Transplantation Guidelines for the management of fungal infections in mechanical circulatory support and cardiothoracic organ transplant recipients: executive summary. J Heart Lung Transpl. 2016;35:261-82.

27. Pelaez T, Munoz P, Guinea J, et al. Outbreak of invasive aspergillosis after major heart surgery caused by spores in the air of the intensive care unit. Clin Infect Dis. 2012;54:e24-31.

28. Hong SK, Hwang S, Lee SG, et al. Pulmonary complications following adult liver transplantation. Transpl Proc. 2006;38:2979-81.

29. Peghin M, Monforte V, Martin-Gomez MT, et al. 10 years of prophylaxis with nebulized liposomal amphotericin B and the changing epidemiology of Aspergillus spp. infection in lung transplantation. Transpl Int. 2016;29:51-62.

30. Munoz P, Ceron I, Valerio M, et al. Invasive aspergillosis among heart transplant recipients: a 24-year perspective. J Heart Lung Transpl. 2014;33:278-88.

31. Prasad A, Agarwal K, Deepak D, et al. Pulmonary aspergillosis: what CT can offer before it is too late! J Clin Diagn Res. 2016;10:TE01-5.

32. Lim C, Seo JB, Park SY, et al. Analysis of initial and follow-up CT findings in patients with invasive pulmonary aspergillosis after solid organ transplantation. Clin Radiol. 2012;67:1179-86.

33. Park SY, Lim C, Lee S-O, et al. Computed tomography findings in invasive pulmonary aspergillosis in non-neutropenic transplant recipients and neutropenic patients, and their prognostic value. J Infect. 2011;63:447-56.

34. Muñoz P, Valerio M, Palomo J, et al. Targeted antifungal prophylaxis in heart transplant recipients. Transplantation. 2013;96:664-9.

35. Stanzani M, Battista G, Sassi C, et al. Computed tomographic pulmonary angiography for diagnosis of invasive mold diseases in patients with hematological malignancies. Clin Infect Dis. 2012;54:610-6.

36. Stanzani M, Sassi C, Lewis RE, et al. High resolution computed tomography angiography improves the radiographic diagnosis of invasive mold disease in patients with hematological malignancies. Clin Infect Dis. 2015;60:1603-10.

37. Henzler C, Henzler T, Buchheidt D, et al. Diagnostic performance of contrast enhanced pulmonary computed tomography angiography for the detection of angioinvasive pulmonary aspergillosis in immunocompromised patients. Sci Rep. 2017;7:4483.

38. Buchheidt D, Reinwald M, Hoenigl M, et al. The evolving landscape of new diagnostic tests for invasive aspergillosis in hematology patients: strengths and weaknesses. Curr Opin Infect Dis. 2017;30:539-44.

39. Escribano P, Marcos-Zambrano LJ, Pelaez T, et al. Sputum and bronchial secretion samples are equally useful as bronchoalveolar 
lavage samples for the diagnosis of invasive pulmonary aspergillosis in selected patients. Med Mycol. 2015;53:235-40.

40. Boch T, Spiess B, Cornely OA, et al. Diagnosis of invasive fungal infections in haematological patients by combined use of galactomannan, 1,3-beta-D-glucan, Aspergillus PCR, multifungal DNA-microarray, and Aspergillus azole resistance PCRs in blood and bronchoalveolar lavage samples: results of a prospective multicentre study. Clin Microbiol Infect. 2016;22:862-8.

41. Giacobbe DR, Del Bono V, Viscoli C, et al. Use of 1,3-betaD-glucan in invasive fungal diseases in hematology patients. Expert Rev Anti Infect Ther. 2017;15:1101-12.

42. Marty FM, Koo S. Role of $(1 \rightarrow 3)$-beta-D-glucan in the diagnosis of invasive aspergillosis. Med Mycol. 2009;47:S233-40.

43. Koo S, Bryar Julie M, Page John H, et al. Diagnostic performance of the $(1 \rightarrow 3)$ beta-D-Glucan assay for Invasive fungal disease. Clin Infect Dis. 2009;49:1650-9.

44. Aslan M, Oz Y, Aksit F, et al. Potential of polymerase chain reaction and galactomannan for the diagnosis of invasive aspergillosis in patients with febrile neutropenia. Mycoses. 2015;58:343-9.

45. Heng SC, Morrissey O, Chen SC, et al. Utility of bronchoalveolar lavage fluid galactomannan alone or in combination with PCR for the diagnosis of invasive aspergillosis in adult hematology patients: a systematic review and meta-analysis. Crit Rev Microbiol. 2015;41:124-34.

46. Oz Y, Aslan M, Aksit F, et al. The effect of clinical characteristics on the performance of galactomannan and PCR for the diagnosis of invasive aspergillosis in febrile neutropenic patients. Mycoses. 2016;59:86-92.

47. Luong ML, Clancy CJ, Vadnerkar A, et al. Comparison of an Aspergillus real-time polymerase chain reaction assay with galactomannan testing of bronchoalvelolar lavage fluid for the diagnosis of invasive pulmonary aspergillosis in lung transplant recipients. Clin Infect Dis. 2011;52:1218-26.

48. Miceli MH, Goggins MI, Chander P, et al. Performance of lateral flow device and galactomannan for the detection of Aspergillus species in bronchoalveolar fluid of patients at risk for invasive pulmonary aspergillosis. Mycoses. 2015;58:368-74.

49. Bergeron A, Porcher R, Menotti J, et al. Prospective evaluation of clinical and biological markers to predict the outcome of invasive pulmonary aspergillosis in hematological patients. J Clin Microbiol. 2012;50:823-30.

50. Zhou W, Li H, Zhang Y, et al. Diagnostic value of galactomannan antigen test in serum and bronchoalveolar lavage fluid samples from patients with nonneutropenic invasive pulmonary aspergillosis. J Clin Microbiol. 2017;55:2153-61.

51. Barnes RA, White PL, Morton CO, et al. Diagnosis of aspergillosis by PCR: clinical considerations and technical tips. Med Mycol. 2018;56:60-72.

52. Buchheidt D, Reinwald M, Hofmann WK, et al. Evaluating the use of PCR for diagnosing invasive aspergillosis. Expert Rev Mol Diagn. 2017;17:603-10.

53. Hummel M, Spiess B, Cornely OA, et al. Aspergillus PCR testing: results from a prospective PCR study within the AmBiLoad trial. Eur J Haematol. 2010;85:164-9.

54. Guinea J, Padilla C, Escribano P, et al. Evaluation of MycAssay Aspergillus for diagnosis of invasive pulmonary aspergillosis in patients without hematological cancer. PLoS One. 2013;8:e61545.

55. Hasseine L, Cassaing S, Robert-Gangneux F, et al. High negative predictive value diagnostic strategies for the reevaluation of early antifungal treatment: a multicenter prospective trial in patients at risk for invasive fungal infections. J Infect. 2015;71:258-65.

56. Johnson GL, Sarker SJ, Nannini F, et al. Aspergillus-specific lateral-flow device and real-time PCR testing of bronchoalveolar lavage fluid: a combination biomarker approach for clinical diagnosis of invasive pulmonary aspergillosis. J Clin Microbiol. 2015;53:2103-8.

57. Pini $\mathrm{P}, \mathrm{Bettua} \mathrm{C}$, Orsi $\mathrm{CF}$, et al. Clinical performance of a commercial real-time PCR assay for Aspergillus DNA detection in serum samples from high-risk patients: comparison with a galactomannan enzyme immunoassay. Eur J Clin Microbiol Infect Dis. 2015;34:131-6.

58. Heinz WJ, Vehreschild JJ, Buchheidt D. Diagnostic work up to assess early response indicators in invasive pulmonary aspergillosis in adult patients with haematologic malignancies. Mycoses. 2019;62:486-93.

59. Perrella A, Esposito C, Amato G, et al. Antifungal prophylaxis with liposomal amphotericin B and caspofungin in high-risk patients after liver transplantation: impact on fungal infections and immune system. Infect Dis. 2016;48:161-6.

60. Perrella A, Esposito C, Pisaniello D, et al. Role of liposomal amphotericin B prophylaxis after liver transplantation compared with fluconazole for high-risk patients. Impact on infections and mortality within one year. Transpl Proc. 2012;44:1977-81.

61. Ben-Ami R, Lewis RE, Kontoyiannis DP. Immunocompromised hosts: immunopharmacology of modern antifungals. Clin Infect Dis. 2008;47:226-35.

62. Ashley ESD, Lewis R, Lewis JS, et al. Pharmacology of systemic antifungal agents. Clin Infect Dis. 2006;43:S28-39.

63. Cornely OA, Arikan-Akdagli S, Dannaoui E, et al. ESCMID and ECMM joint clinical guidelines for the diagnosis and management of mucormycosis 2013. Clin Microbiol Infect. 2014;20:5-26.

64. Garcia-Vidal C. Opciones terapeuticas actuales en las micosis invasoras y papel terapeutico potencial del isavuconazol [Current therapeutic options in invasive mycosis and potential therapeutic role of isavuconazole]. Rev Iberoam Micol. 2018;35:192-7.

65. Sipsas NV, Gamaletsou MN, Anastasopoulou A, et al. Therapy of mucormycosis. J Fungi. 2018;4:E90.

66. Cornely OA, Alastruey-Izquierdo A, Arenz D, et al. Global guideline for the diagnosis and management of mucormycosis: an initiative of the ECMM in cooperation with the MSG ERC. HemaSphere. 2019;3:588.

67. Tortorano AM, Richardson M, Roilides E, et al. ESCMID and ECMM joint guidelines on diagnosis and management of hyalohyphomycosis: Fusarium spp., Scedosporium spp. and others. Clin Microbiol Infect. 2014;20:27-46.

68. Maertens JA, Raad II, Marr KA, et al. Isavuconazole versus voriconazole for primary treatment of invasive mould disease caused by Aspergillus and other filamentous fungi (SECURE): a phase 3, randomised-controlled, non-inferiority trial. Lancet. 2016;387:760-9.

69. Li J, Nguyen CT, Garcia-Diaz J. Role of new antifungal agents in the treatment of invasive fungal infections in transplant recipients: isavuconazole and new posaconazole formulations. J Fungi. 2015;1:345-66.

70. Aguilar C, Husain S, Lortholary O. Recent advances in understanding and managing infectious diseases in solid organ transplant recipients. F1000Res. 2018. https://doi.org/10.12688/f1000 research.14262.1.

71. Jenks JD, Reed SL, Seidel D, et al. Rare mould infections caused by Mucorales, Lomentospora prolificans and Fusarium, in San Diego, CA: the role of antifungal combination therapy. Int J Antimicrob Agents. 2018;52:706-12.

72. Morales P, Galán G, SanMartín E, et al. Intrabronchial instillation of amphotericin B lipid complex: a case report. Transpl Proc. 2009;41:2223-4.

73. Segal BH, Herbrecht R, Stevens DA, et al. Defining responses to therapy and study outcomes in clinical trials of invasive fungal diseases: Mycoses Study Group and European Organization for 
Research and Treatment of Cancer consensus criteria. Clin Infect Dis. 2008;47:674-83.

74. Guinea J, Bouza E. Current challenges in the microbiological diagnosis of invasive aspergillosis. Mycopathologia. 2014;178:403-16.

75. Brodöfel H, Vogel M, Hebart H, et al. Long-term CT followup in 40 non-HIV immunocompromised patients with invasive pulmonary aspergillosis: kinetics of CT morphology and correlation with clinical findings and outcome. Am J Roentgenol. 2006;187:404-13.

76. Caillot D, Latrabe V, Thiebaut A, et al. Computer tomography in pulmonary invasive aspergillosis in hematological patients with neutropenia: an useful tool for diagnosis and assessment of outcome in clinical trials. Eur J Radiol. 2010;74:e172-5.

77. Vehreschild JJ, Heussel CP, Groll AH, et al. Serial assessment of pulmonary lesion volume by computed tomography allows survival prediction in invasive pulmonary aspergillosis. Eur Radiol. 2017;27:3275-82.

78. Jaijakul S, Vazquez JA, Swanson RN, et al. (1,3)-beta-D-glucan as a prognostic marker of treatment response in invasive candidiasis. Clin Infect Dis. 2012;55:521-6.

79. Chai LY, Kullberg BJ, Johnson EM, et al. Early serum galactomannan trend as a predictor of outcome of invasive aspergillosis. J Clin Microbiol. 2012;50:2330-6.

80. Fisher CE, Stevens AM, Leisenring W, et al. The serum galactomannan index predicts mortality in hematopoietic stem cell transplant recipients with invasive aspergillosis. Clin Infect Dis. 2013;57:1001-4.

81. Han SB, Kim SK, Lee JW, et al. Serum galactomannan index for early prediction of mortality in immunocompromised children with invasive pulmonary aspergillosis. BMC Infect Dis. 2015;15:271.

82. Kovanda LL, Kolamunnage-Dona R, Neely M, et al. Pharmacodynamics of isavuconazole for invasive mold disease: role of galactomannan for real-time monitoring of therapeutic response. Clin Infect Dis. 2017;64:1557-63.

83. McCarthy MW, Walsh TJ. Galactomannan antigenemia as a biomarker for therapeutic response of invasive aspergillosis: implications for clinical trial design and patient care. Expert Rev Mol Diagn. 2018;18:601-4.

84. Miceli MH, Grazziutti ML, Woods G, et al. Strong correlation between serum aspergillus galactomannan index and outcome of aspergillosis in patients with hematological cancer: clinical and research implications. Clin Infect Dis. 2008;46:1412-22.

85. Russo A, Giuliano S, Vena A, et al. Predictors of mortality in non-neutropenic patients with invasive pulmonary aspergillosis: does galactomannan have a role? Diagn Microbiol Infect Dis. 2014;80:83-6.

86. Lopez-Medrano F, Fernandez-Ruiz M, Silva JT, et al. Clinical presentation and determinants of mortality of invasive pulmonary aspergillosis in kidney transplant recipients: a multinational cohort study. Am J Transpl. 2016;16:3220-34.

87. Chai L, Netea MG, Teerenstra S, et al. Early proinflammatory cytokines and C-reactive protein trends as predictors of outcome in invasive aspergillosis. J Infect Dis. 2010;202:1454-62.

88. Cho HJ, Jang MS, Hong SD, et al. Prognostic factors for survival in patients with acute invasive fungal rhinosinusitis. Am J Rhinol Allergy. 2015;29:48-53.

89. Krel M, Petraitis V, Petraitiene R, et al. Host biomarkers of invasive pulmonary aspergillosis to monitor therapeutic response. Antimicrob Agents Chemother. 2014;58:3373-8.

90. Roques M, Chretien ML, Favennec C, et al. Evolution of procalcitonin, C-reactive protein and fibrinogen levels in neutropenic leukaemia patients with invasive pulmonary aspergillosis or mucormycosis. Mycoses. 2016;59:383-90.

91. Zhao Y, Nagasaki Y, Paderu P, et al. Applying host disease status biomarkers to therapeutic response monitoring in invasive aspergillosis patients. Med Mycol. 2019;57:38-44.

92. Bellocchio S, Gaziano R, Bozza S, et al. Liposomal amphotericin $B$ activates antifungal resistance with reduced toxicity by diverting Toll-like receptor signalling from TLR-2 to TLR-4. J Antimicrob Chemother. 2005;55:214-22.

93. Lewis RE, Chamilos G, Prince RA, et al. Pretreatment with empty liposomes attenuates the immunopathology of invasive pulmonary aspergillosis in corticosteroid-immunosuppressed mice. Antimicrob Agents Chemother. 2007;51:1078-81.

94. Walker L, Sood P, Lenardon MD, et al. The viscoelastic properties of the fungal cell wall allow traffic of Am Bisome as intact liposome vesicles. mBio. 2018. https://doi.org/10.1128/ mBio.02383-17.

95. Ambati S, Ferarro AR, Khang SE, et al. Dectin-1-targeted antifungal liposomes exhibit enhanced efficacy. mSphere. 2019. https ://doi.org/10.1128/mSphere.00025-19.

96. Davies GR, Hope W, Khoo S. Opinion: the pharmacometrics of infectious disease. CPT Pharmacomet Syst Pharmacol. 2013;28:e70.

97. Zhang S, Cai S, Ma Y. Association between Fusobacterium nucleatum and colorectal cancer: progress and future directions. J Cancer. 2018;9:1652-9.

98. Romani L, Zelante T, De Luca A, et al. Microbiota control of a tryptophan-AhR pathway in disease tolerance to fungi. Eur J Immunol. 2014;44:3192-200.

99. Romani L, Zelante T, Palmieri M, et al. The cross-talk between opportunistic fungi and the mammalian host via microbiota's metabolism. Semin Immunopathol. 2015;37:163-71.

100. Chai LY, Netea MG, Tai BC, et al. An elevated pro-inflammatory cytokine response is linked to development of amphotericin B-induced nephrotoxicity. J Antimicrob Chemother. 2013;68:1655-9. 